\title{
On anti-structurable algebras and extended Dynkin diagrams
}

\author{
Noriaki KAMIYA ${ }^{a}$ and Daniel MONDOC ${ }^{b}$ \\ ${ }^{a}$ Center for Mathematical Sciences, University of Aizu, Aizuwakamatsu, \\ 965-8580 Fukushima, Japan \\ ${ }^{b}$ Kungliga Tekniska högskolan, Valhallavägen 79, 10044 Stockholm, Sweden \\ E-mails:kamiya@u-aizu.ac.jp, mondoc@math.kth.se
}

\begin{abstract}
We construct Lie superalgebras $\mathfrak{o s p}(2 n+1 \mid 4 n+2)$ and $\mathfrak{o s p}(2 n \mid 4 n)$ starting with certain classes of anti-structurable algebras via the standard embedding Lie superalgebra construction corresponding to $(\epsilon, \delta)$-Freudenthal Kantor triple systems.
\end{abstract}

AMS MSC 2000: 17A30, 17B60

\section{Introduction}

1.1 $(\epsilon, \delta)$-Freudenthal Kantor triple systems, $\delta$-Lie triple systems, and Lie (super)algebras

We are concerned in this paper with triple systems which have finite dimension over a field $\Phi$ of characteristic $\neq 2$ or 3 , unless otherwise specified.

In order to render this paper as self-contained as possible, we recall first the definition of a generalized Jordan triple system of second order (for short GJTS of 2nd order).

Definition 1.1. A vector space $V$ over a field $\Phi$ endowed with a trilinear operation $V \times$ $V \times V \rightarrow V,(x, y, z) \longmapsto(x y z)$ is said to be a GJTS of 2nd order if the following conditions are fulfilled:

$$
\begin{aligned}
& (a b(x y z))=((a b x) y z)-(x(b a y) z)+(x y(a b z)) \\
& K(K(a, b) x, y)-L(y, x) K(a, b)-K(a, b) L(x, y)=0
\end{aligned}
$$

where

$$
L(a, b) c:=(a b c) \quad \text { and } \quad K(a, b) c:=(a c b)-(b c a)
$$

Definition 1.2. A Jordan triple system (for short JTS) satisfies (1.1) and $(a b c)=(c b a)$, $\forall a, b, c \in V$.

We can generalize the concept of GJTS of the 2nd order as follows (see $[10,11,13,15,32]$ ).

Definition 1.3. For $\varepsilon= \pm 1$ and $\delta= \pm 1$, a triple product that satisfies the identities

$$
\begin{aligned}
& (a b(x y z))=((a b x) y z)+\varepsilon(x(b a y) z)+(x y(a b z)) \\
& K(K(a, b) x, y)-L(y, x) K(a, b)+\varepsilon K(a, b) L(x, y)=0
\end{aligned}
$$

where

$$
L(a, b) c:=(a b c), \quad K(a, b) c:=(a c b)-\delta(b c a)
$$

is called an $(\varepsilon, \delta)$-Freudenthal-Kantor triple system (for short $(\varepsilon, \delta)$-FKTS). 
Remark 1.4. Note that $K(b, a)=-\delta K(a, b)$.

Definition 1.5. An $(\varepsilon, \delta)$-FKTS $U$ is called unitary if the identity map $I d$ is contained in $\kappa:=K(U, U)$, i.e., if there exist $a_{i}, b_{i} \in U$ such that $\Sigma_{i} K\left(a_{i}, b_{i}\right)=I d$.

Let $U$ be an $(\varepsilon, \delta)$-FKTS and let $V_{k}, k=1,2,3$, be subspaces of $U$. We denote by $\left(V_{1}, V_{2}, V_{3}\right)$ the subspace of $U$ spanned by elements $\left(x_{1}, x_{2}, x_{3}\right), x_{k} \in V_{k}, k=1,2,3$.

Definition 1.6. A subspace $V$ of $U$ is called an ideal of an $(\varepsilon, \delta)$-FKTS $U$ if the following relations hold: $(V, U, U) \subseteq V,(U, V, U) \subseteq V,(U, U, V) \subseteq V$. $U$ is called simple if $(,$,$) is$ not a zero map and $U$ has no nontrivial ideal.

We denote the triple products by $(x y z),\{x y z\},[x y z]$, and $\langle x y z\rangle$ upon their suitability.

Remark 1.7. We note that the concept of GJTS of 2 nd order coincides with that of $(-1,1)$ FKTS. Thus we can construct the simple Lie algebras by means of the standard embedding method (see $[5,10,11,12,13,15,16,17,22,32]$ ).

Remark 1.8. We note that the two pairs of identities (1.3-1.4) and (1.6) are equivalent

$$
\begin{aligned}
& {[L(a, b), L(x, y)]=L((a b x), y)+\varepsilon L(x,(b a y))} \\
& K(K(a, b) x, y)-K((y x a), b)-K(a,(y x b))=0
\end{aligned}
$$

where $\varepsilon= \pm 1, \delta= \pm 1$ and $L(a, b), K(a, b)$ are defined by (1.5).

Indeed, from (1.3) and (1.4) follows (1.6b). Conversely, from (1.6a) and (1.6b) it follows that (1.4) holds.

For an $(\varepsilon, \delta)$-FKTS $U$, we denote

$$
S(a, b):=L(a, b)+\varepsilon L(b, a), \quad A(a, b):=L(a, b)-\varepsilon L(b, a)
$$

where $L(a, b)$ is defined by $(1.5)$.

Remark 1.9. We note that $S(a, b)=\varepsilon S(b, a)$.

Then $S(a, b)$ (resp., $A(a, b)$ ) is a derivation (resp., anti-derivation) of $U$.

Indeed, we note that the identities (1.7) and (1.8) are valid.

$$
\begin{aligned}
& {[S(a, b), L(c, d)]=L(S(a, b) c, d)+L(c, S(a, b) d)} \\
& {[A(a, b), L(c, d)]=L(A(a, b) c, d)-L(c, A(a, b) d)}
\end{aligned}
$$

Definition 1.10. For $\delta= \pm 1$, a triple system $(a, b, c) \mapsto[a b c], a, b, c \in V$ is called a $\delta$-Lie triple system (for short $\delta$-LTS) if the following identities are fulfilled:

$$
\begin{aligned}
& {[a b c]=-\delta[b a c]} \\
& {[a b c]+[b c a]+[c a b]=0} \\
& {[a b[x y z]]=[[a b x] y z]+[x[a b y] z]+[x y[a b z]]}
\end{aligned}
$$

where $a, b, x, y, z \in V$. An 1-LTS is a LTS, while a -1-LTS is called an anti-LTS, by [11]. 
Proposition 1.11 (see $[11,15])$. Let $U(\varepsilon, \delta)$ be an $(\varepsilon, \delta)$-FKTS. If $J$ is an endomorphism of $U(\varepsilon, \delta)$ such that $J\langle x y z\rangle=\langle J x J y J z\rangle$ and $J^{2}=-\varepsilon \delta I d$, then $(U(\varepsilon, \delta),[x y z])$ is an LTS (if $\delta=1$ ) or an anti-LTS (if $\delta=-1$ ) with respect to the product (1.9):

$$
[x y z]:=\langle x J y z\rangle-\delta\langle y J x z\rangle+\delta\langle x J z y\rangle-\langle y J z x\rangle
$$

Corollary 1.12. Let $U(\varepsilon, \delta)$ be an $(\varepsilon, \delta)$-FKTS. Then the vector space $T(\varepsilon, \delta)=U(\varepsilon, \delta) \oplus$ $U(\varepsilon, \delta)$ becomes an LTS (if $\delta=1$ ) or an anti-LTS (if $\delta=-1$ ) with respect to the triple product (1.10):

$$
\left[\left(\begin{array}{l}
a \\
b
\end{array}\right)\left(\begin{array}{l}
c \\
d
\end{array}\right)\left(\begin{array}{l}
e \\
f
\end{array}\right)\right]=\left(\begin{array}{cc}
L(a, d)-\delta L(c, b) & \delta K(a, c) \\
-\varepsilon K(b, d) & \varepsilon(L(d, a)-\delta L(b, c))
\end{array}\right)\left(\begin{array}{l}
e \\
f
\end{array}\right)
$$

Remark 1.13. Thus we can obtain the standard embedding Lie algebra (if $\delta=1$ ) or Lie superalgebra (if $\delta=-1$ ), $L(\varepsilon, \delta)=D(T(\varepsilon, \delta), T(\varepsilon, \delta)) \oplus T(\varepsilon, \delta)$, associated to $T(\varepsilon, \delta)$, where $D(T(\varepsilon, \delta), T(\varepsilon, \delta))$ is the set of inner derivations of $T(\varepsilon, \delta)$, i.e.,

$$
\begin{aligned}
D(T(\varepsilon, \delta), T(\varepsilon, \delta)) & :=\left\{\left(\begin{array}{cc}
L(a, b) & \delta K(c, d) \\
-\varepsilon K(e, f) & \varepsilon L(b, a)
\end{array}\right)\right\}_{\mathrm{span}} \\
T(\varepsilon, \delta) & :=\left\{\left(\begin{array}{l}
x \\
y
\end{array}\right) \mid x, y \in U(\varepsilon, \delta)\right\}_{\mathrm{span}}
\end{aligned}
$$

Remark 1.14. $L(\varepsilon, \delta)=L_{-2} \oplus L_{-1} \oplus L_{0} \oplus L_{1} \oplus L_{2}$ is the 5-graded Lie (super)algebra such that $L_{-1} \oplus L_{1}=T(\varepsilon, \delta), D(T(\varepsilon, \delta), T(\varepsilon, \delta))=L_{-2} \oplus L_{0} \oplus L_{2}$, and $\left[L_{i}, L_{j}\right] \subseteq L_{i+j}$. This Lie (super)algebra construction is one of the reasons to study nonassociative algebras and triple systems.

\section{$1.2 \delta$-structurable algebras}

The existence of the class of nonassociative algebras called structurable algebras is an important generalization of Jordan algebras giving a construction of Lie algebras. Hence from our concept, by means of triple products, we define a generalization of such class to construct Lie superalgebras as well as Lie algebras. Our start point briefly described in a historical setting is the construction of Lie (super)algebras starting from a class of nonassociative algebras. Hence within the general framework of $(\epsilon, \delta)$-FKTSs $(\epsilon, \delta= \pm 1)$ and the standard embedding Lie (super)algebra construction studied in $[5,6,10,11,12,17]$ (see also references therein) we defined $\delta$-structurable algebras (see [18]) as a class of nonassociative algebras with involution which coincides with the class of structurable algebras for $\delta=1$ as introduced and studied in $[1,2]$. Structurable algebras are a class of nonassociative algebras with involution that include Jordan algebras (with trivial involution), associative algebras with involution, and alternative algebras with involution. They are related to GJTSs of 2 nd order, or $(-1,1)$-FKTSs, as introduced and studied in [20,21] and further studied in [3, 4, 19, 26, 27, 28, 29, 30] (see also references therein). Their importance lies with constructions of 5-graded Lie algebras $L(\epsilon, \delta)=L_{-2} \oplus L_{-1} \oplus L_{0} \oplus L_{1} \oplus L_{2},\left[L_{i}, L_{j}\right] \subseteq L_{i+j}$. For $\delta=-1$, the anti-structurable algebras (see [18]) are a class of nonassociative algebras that may similarly shed light on the notion of $(-1,-1)$-FKTSs hence, by $[5,6]$, on the construction of Lie superalgebras and Jordan algebras as it will be shown.

Throughout the paper, it is assumed that $\left(\mathcal{A},{ }^{-}\right)$is a finite-dimensional nonassociative unital algebra with involution (involutive anti-automorphism, i.e., $\overline{\bar{x}}=x$ and $\overline{x y}=\bar{y} \bar{x}$ for $x, y \in \mathcal{A}$ ) over $\Phi$. The identity element of $\mathcal{A}$ is denoted by 1 . 
Remark 1.15. By [1] we have $\mathcal{A}=\mathcal{H} \oplus \mathcal{S}$, where $\mathcal{H}=\{a \in \mathcal{A} \mid \bar{a}=a\}$ and $\mathcal{S}=\{a \in \mathcal{A} \mid \bar{a}=-a\}$.

Suppose $x, y, z \in \mathcal{A}$. Put $[x, y]:=x y-y x,[x, y, z]:=(x y) z-x(y z)$. Note that (1.11) is valid.

$$
\overline{[x, y, z]}=-[\bar{z}, \bar{y}, \bar{x}]
$$

Let $L_{x}, R_{x}$ be defined by $L_{x}(y):=x y, R_{x}(y):=y x, x, y \in \mathcal{A}$. For $\delta= \pm 1$, define (1.12) and (1.13).

$$
\begin{aligned}
& { }^{\delta} V_{x, y}:=L_{L_{x}(\bar{y})}+\delta\left(R_{x} R_{\bar{y}}-R_{y} R_{\bar{x}}\right) \\
& { }^{\delta} B_{\mathcal{A}}(x, y, z):={ }^{\delta} V_{x, y}(z)=(x \bar{y}) z+\delta[(z \bar{y}) x-(z \bar{x}) y], \quad x, y, z \in \mathcal{A}
\end{aligned}
$$

Definition 1.16. ${ }^{+} B_{\mathcal{A}}(x, y, z)$ is called the triple system obtained from the algebra $\left(\mathcal{A},{ }^{-}\right)$. We call ${ }^{-} B_{\mathcal{A}}(x, y, z)$ the anti-triple system obtained from the algebra $\left(\mathcal{A},^{-}\right)$.

We will write for short $V_{x, y}:={ }^{\delta} V_{x, y}, B_{\mathcal{A}}:=\left({ }^{\delta} B_{\mathcal{A}}, \mathcal{A}\right)$.

Remark 1.17. The upper left index notation is chosen in order not to be mixed with the upper right index notation of [1] which has a different meaning.

Definition 1.18. A unital nonassociative algebra with involution $\left(\mathcal{A},,^{-}\right)$is called a structurable algebra if the following identity is fulfilled:

$$
\left[V_{u, v}, V_{x, y}\right]=V_{V_{u, v}(x), y}-V_{x, V_{v, u}(y)}
$$

for $V_{u, v}={ }^{+} V_{u, v}, V_{x, y}={ }^{+} V_{x, y}, u, v, x, y \in \mathcal{A}$, and we will call $\left(\mathcal{A},{ }^{-}\right)$an anti-structurable algebra if identity (1.14) is fulfilled for $V_{u, v}={ }^{-} V_{u, v}, V_{x, y}={ }^{-} V_{x, y}$.

Remark 1.19. If $\left(\mathcal{A},{ }^{-}\right)$is structurable, then, in the terminology of [21], the triple system $B_{\mathcal{A}}$ is called a GJTS and by [7], $B_{\mathcal{A}}$ is a GJTS of 2 nd order, i.e., satisfies the identities (1.3) and (1.4).

Definition 1.20. If $\left(\mathcal{A},{ }^{-}\right)$is anti-structurable, then we call $B_{\mathcal{A}}$ an anti-GJTS.

Put $T_{x}:=V_{x, 1}$ for $x \in \mathcal{A}$. Then, by (1.12), $T_{x}=L_{x}+\delta R_{x-\bar{x}}$ for $x \in \mathcal{A}$, thus $T_{h}=L_{h}$, $h \in \mathcal{H}$.

Remark 1.21. (i) If $u=h \in \mathcal{H}$ and $x, y \in \mathcal{A}$, (1.14) becomes (1.15).

$$
\left[L_{h}, V_{x, y}\right]=V_{h x, y}-V_{x, h y}
$$

(ii) Suppose ${ }^{-}$is the identity map and hence $\mathcal{A}$ is commutative. If $\left(\mathcal{A},{ }^{-}\right)$is $\delta$-structurable, then $\mathcal{A}$ is a Jordan algebra, by [18]. Conversely, by [24, Section 3], any Jordan algebra satisfies (1.15) if $V_{x, y}={ }^{+} V_{x, y}$ for $x, y \in \mathcal{A}$, hence it is structurable. By (1.15) and [18], any Jordan algebra is anti-structurable if it satisfies $((h x) y) z-h((x y) z)=(x(y h)) z-(x y)(h z)$ for $h, x, y, z \in \mathcal{A}$.

Clearly, the last identity is fulfilled by an associative algebra.

(iii) If $x \in \mathcal{A}$ and $T_{x}(1)=0$, then $x=0$, by [18].

Definition 1.22. For $s \in \mathcal{S}$ and $h \in \mathcal{H}$, we say that $\left(\mathcal{A},{ }^{-}\right)$is $\mathcal{S}$ skew-alternative if $[s, x, y]=$ $-[x, s, y]$ while $(\mathcal{A},-)$ is $\mathcal{H}$ skew-alternative if $[h, x, y]=-[x, h, y]$ for $x, y \in \mathcal{A}$. 
Remark 1.23. If $\left(\mathcal{A},{ }^{-}\right)$is $\mathcal{S}$ skew-alternative, then by $[1],[s, x, y]=-[x, s, y]=[x, y, s], s \in$ $\mathcal{S}, x, y \in \mathcal{A}$. If $\left(\mathcal{A},{ }^{-}\right)$is $\mathcal{H}$ skew-alternative, then by $(1.11),[h, x, y]=-[x, h, y]=[x, y, h]$, $h \in \mathcal{H}, x, y \in \mathcal{A}$.

Proposition 1.24 (see [18]). If $\left(\mathcal{A},^{-}\right)$is structurable, then $\left(\mathcal{A},{ }^{-}\right)$is $\mathcal{S}$ skew-alternative. If $\left(\mathcal{A},{ }^{-}\right)$is anti-structurable, then $\left(\mathcal{A},^{-}\right)$is $\mathcal{H}$ skew-alternative.

Remark 1.25. Let $\left(\mathcal{A},^{-}\right)$be a $\delta$-structurable algebra and let $\operatorname{Der}\left(\mathcal{A},{ }^{-}\right)$be the set of derivations of $\mathcal{A}$ that commute with ${ }^{-}$. By Remark (iii) above $T_{\mathcal{A}} \cap \operatorname{Der}\left(\mathcal{A},{ }^{-}\right)=0$ and so we may define the structure algebra $\operatorname{Str}\left(\mathcal{A},{ }^{-}\right):=T_{\mathcal{A}} \oplus \operatorname{Der}\left(\mathcal{A},{ }^{-}\right)$. This algebra plays an important role in the structure study of structurable algebras (see [1]) and may play a role in the structure study of anti-structurable algebras (theory to be presented elsewhere).

\section{$1.3 \quad$ Examples}

For examples of structurable algebras, we refer to $[1,2]$.

Definition 1.26. Let $(B, U)$ and $\left(B^{\prime}, U^{\prime}\right)$ be two triple systems. A linear map $\mu$ of $U$ into $U^{\prime}$ is called a homomorphism if $\mu$ satisfies $\mu(B(x, y, z))=B^{\prime}(\mu(x), \mu(y), \mu(z)), x, y, z \in U$. Moreover, if $\mu$ is bijective, then $\mu$ is called an isomorphism and $(B, U)$ and $\left(B^{\prime}, U^{\prime}\right)$ are said to be isomorphic.

Definition 1.27. Let $\left(A,^{-}\right)$be a unital nonassociative algebra over $\Phi$ with involution and let $\left(A^{o p},-\right)$ denote the opposite algebra, i.e., the algebra with multiplication defined by $x{ }_{o p} y=y x, x, y \in A$, where in the right-hand side of the equality the multiplication is done in $A$.

Remark 1.28. The algebras $\left(A,^{-}\right)$and $\left(A^{o p},-\right)$ are isomorphic under the map $x \mapsto \bar{x}$.

Let $L_{x}, R_{x}$ be defined by $L_{x}(y):=x y, R_{x}(y):=y x, x, y \in \mathcal{A}$. For $\delta= \pm 1$, define $(1.16)$ and (1.17).

$$
\begin{aligned}
& { }^{\delta} V_{x, y}^{o p}:=R_{R_{x}(\bar{y})}+\delta\left(L_{x} L_{\bar{y}}-L_{y} L_{\bar{x}}\right) \\
& { }^{\delta} B_{\mathcal{A}}^{o p}(x, y, z):={ }^{\delta} V_{x, y}^{o p}(z)=z(\bar{y} x)+\delta[x(\bar{y} z)-y(\bar{x} z)], \quad x, y, z \in \mathcal{A}
\end{aligned}
$$

Proposition 1.29. $\mathcal{A}$ is a $\delta$-structurable algebra if and only if $\mathcal{A}^{o p}$ is a $\delta$-structurable algebra.

Proof. Clearly, $B_{A}^{o p}$ is the triple system obtained from the algebra $\left(\mathcal{A}^{o p},-\right)$, and so $B_{\mathcal{A}}$ and $B_{\mathcal{A}}^{o p}$ are isomorphic under the map $x \mapsto \bar{x}$, by (1.13) and (1.17).

Let $\mathcal{M}_{m, n}(\Phi)$ denote the vector space of $m \times n$ matrices over $\Phi$ and for $x \in \mathcal{M}_{m, n}(\Phi)$ denote by $x^{\top}$ the transposed matrix.

Lemma 1.30. $\left(\mathcal{M}_{m, n}(\Phi),\{x, y, z\}\right)$ is a $(-1, \delta)$-FKTS, where $\{x, y, z\}$ is defined by (1.18).

$$
\{x, y, z\}:=x y^{\top} z+\delta\left(z y^{\top} x-z x^{\top} y\right), \quad x, y, z \in \mathcal{M}_{m, n}(\Phi)
$$

Proof. It is straightforward calculation to show that the identities (1.3) and (1.4) hold.

Theorem 1.31. $\mathcal{M}_{n, n}(\Phi)$ with the involution $x \mapsto x^{\top}$ is a $\delta$-structurable algebra.

Proof. It is a direct consequence of Lemma 1.30.

Example 1.32. $\left(\mathcal{M}_{m, n}(\mathbb{C}),\{x, y, z\}\right)$ is a $(-1, \delta)$-FKTS, where $\{x, y, z\}$ is defined by (1.19)

$$
\{x, y, z\}:=x \bar{y}^{\top} z+\delta\left(z \bar{y}^{\top} x-z \bar{x}^{\top} y\right), \quad x, y, z \in \mathcal{M}_{m, n}(\mathbb{C})
$$


Indeed, it is straightforward calculation to show that the identities (1.3) and (1.4) hold. Hence $\mathcal{M}_{n, n}(\mathbf{C})$ with the involution $x \mapsto \bar{x}^{\top}$ is a $\delta$-structurable algebra.

Remark 1.33. By [17], the following construction of Lie superalgebras is obtained by the standard embedding method. If $U(-1,-1):=\mathcal{M}_{2 n, m}(\Phi)$ with the product $(1.18)$, then the corresponding standard embedding Lie superalgebra is $\mathfrak{o s p}(2 n \mid 2 m)=D(n, m)$ (as defined by $[8,9])$, hence the standard embedding Lie superalgebra of the anti-structurable algebra $\mathcal{M}_{2 n, 2 n}(\Phi)$ is $\mathfrak{o s p}(2 n \mid 4 n)$. Similarly, if $U(-1,-1):=\mathcal{M}_{2 n+1, m}(\Phi)$ with the product (1.18), then the corresponding standard embedding Lie superalgebra is osp $(2 n+1 \mid 2 m)=B(n, m)$ (as defined by $[8,9]$ ), hence the standard embedding Lie superalgebra of the anti-structurable algebra $\mathcal{M}_{2 n+1,2 n+1}(\Phi)$ is osp $(2 n+1 \mid 4 n+2)$.

The construction of these Lie superalgebras and the correspondence with extended Dynkin diagrams is the subject of the next section. The study of the structure theory of antistructurable algebras, the Peirce decomposition (as defined by [14, 23]), will be considered as future work. Moreover, let $\mathrm{U}$ be an anti-structurable algebra and associative algebra, then $\mathrm{U}$ is a $(-1,-1)$-FKTS. The details will be described in a future paper.

\section{Anti-structurable algebras and extended Dynkin diagrams}

Let $U:=\mathcal{M}_{l, l}(\Phi)$ with the product (1.18) and $\delta=-1$, that is, $\{x, y, z\}$ is defined by (2.1)

$$
\{x, y, z\}:=x y^{\top} z-z y^{\top} x+z x^{\top} y, \quad x, y, z \in \mathcal{M}_{l, l}(\Phi)
$$

Then from the previous section this triple system is a simple unitary $(-1,-1)$-FKTS obtained from anti-structurable algebra $\left(U,^{\top}\right)$. Hence by the methods of the standard embedding associated to $U$ we can obtain the standard embedding Lie superalgebra as follows from the following proposition: the Lie (super)algebras notations and extended Dynkin diagrams are those of [8].

Proposition 2.1. Let $\left(U,^{\top}\right), U:=\mathcal{M}_{l, l}(\Phi)$ be anti-structurable algebras and let $L(U)=$ $\oplus_{l=-2}^{2} L_{l}$ be the standard embedding Lie superalgebra. Then $L(U), L_{-2} \oplus L_{0} \oplus L_{2}, L_{0}$ and the corresponding extended Dynkin diagrams with $\otimes$ roots deleted are

$$
\begin{aligned}
& \text { (i) }\left\{\begin{array}{l}
L(U)=B(n, l)=\mathfrak{o s p}(l \mid 2 l) \\
L_{-2} \oplus L_{0} \oplus L_{2}=C_{l} \oplus B_{n}, \quad \text { for } l=2 n+1 \\
L_{0}=A_{l-1} \oplus B_{n} \oplus \lambda \Phi
\end{array}\right.
\end{aligned}
$$

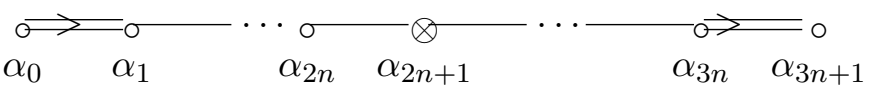

$$
\begin{aligned}
& \text { (ii) }\left\{\begin{array}{l}
L(U)=D(n, l)=\mathfrak{o s p}(l \mid 2 l) \\
L_{-2} \oplus L_{0} \oplus L_{2}=C_{l} \oplus D_{n}, \quad \text { for } l=2 n \\
L_{0}=A_{l-1} \oplus D_{n} \oplus \lambda \Phi
\end{array}\right.
\end{aligned}
$$

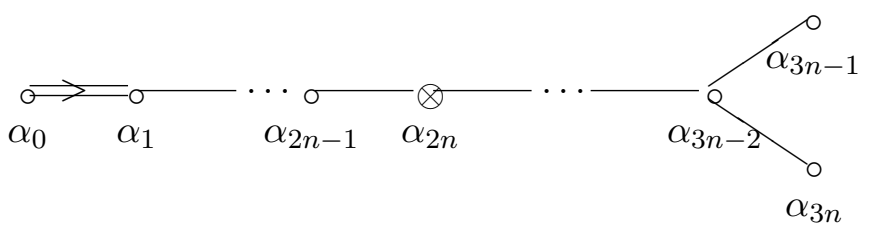


Proof. From $\kappa=\left\{x^{\top} z+z^{\top} x\right\}_{\text {span }}=\left\{A \mid A^{\top}=A, A \in \mathcal{M}_{l, l}(\Phi)\right\}$ and somewhat long calculations, it follows that $\left(U:=\mathcal{M}_{l, l}(\Phi),\{\}\right)$ defined by $(2.1)$ are simple unitary $(-1,-1)$ FKTSs. Then the standard embedding Lie superalgebras follows from [17]. Moreover, since $L_{\overline{1}}=L_{-1} \oplus L_{1}$ is an anti-LTS and $L_{\overline{0}}=L_{-2} \oplus L_{0} \oplus L_{1}=\operatorname{Der}\left(L_{-1} \oplus L_{1}\right)$, it is a straightforward calculation to check that $L_{\overline{0}}$ is obtained from the extended Dynkin diagram of $L(U)$ by deleting the root $\alpha_{l}$, while $L_{0}$ is isomorphic to the corresponding Dynkin diagram ( $\alpha_{l}$ deleted) $\oplus \lambda \Phi$.

Remark 2.2. These results mean that the correspondence between anti-structurable algebras and extended Dynkin diagrams is a useful concept for the structure theory of triple systems.

\section{References}

[1] B. N. Allison. A class of nonassociative algebras with involution containing the class of Jordan algebras. Math. Ann., 237 (1978), 133-156.

[2] B. N. Allison. Models of isotropic simple Lie algebras. Comm. Algebra, 7 (1979), 1835-1875.

[3] H. Asano and K. Yamaguti. A construction of Lie algebras by generalized Jordan triple systems of second order. Nederl. Akad. Wetensch. Indag. Math., 42 (1980), 249-253.

[4] H. Asano. Classification of non-compact real simple generalized Jordan triple systems of the second kind. Hiroshima Math. J., 21 (1991), 463-489.

[5] A. Elduque, N. Kamiya, and S. Okubo. Simple $(-1,-1)$ balanced Freudenthal Kantor triple systems. Glasg. Math. J., 11 (2003), 353-372.

[6] A. Elduque, N. Kamiya, and S. Okubo. $(-1,-1)$ balanced Freudenthal Kantor triple systems and noncommutative Jordan algebras. J. Algebra, 294 (2005), 19-40.

[7] J. R. Faulkner. Structurable triples, Lie triples, and symmetric spaces. Forum Math., 6 (1994), 637-650.

[8] L. Frappat, A. Sciarrino, and P. Sorba. Dictionary on Lie Algebras and Superalgebras. Academic Press, San Diego, California, 2000.

[9] V. G. Kac. Lie superalgebras. Adv. Math., 26 (1977), 8-96.

[10] N. Kamiya. A structure theory of Freudenthal-Kantor triple systems. J. Algebra, 110 (1987), $108-123$.

[11] N. Kamiya. A construction of anti-Lie triple systems from a class of triple systems. Mem. Fac. Sci. Shimane Univ., 22 (1988), 51-62.

[12] N. Kamiya. A structure theory of Freudenthal-Kantor triple systems. II. Comment. Math. Univ. St. Paul., 38 (1989), 41-60.

[13] N. Kamiya. The construction of all simple Lie algebras over $\mathbb{C}$ from balanced Freudenthal-Kantor triple systems. In "Contributions to General Algebra, 7". D. Dorninger, G. Eigenthaler, H. K. Kaiser, and W. B. Müller, Eds. Hölder-Pichler-Tempsky, Vienna, 1991, 205-213.

[14] N. Kamiya. Examples of Peirce decomposition of generalized Jordan triple system of second order-Balanced cases. In "Noncommutative Geometry and Representation Theory in Mathematical Physics". J. Fuchs, J. Michelson, G. Rozenblioum, A. Stolin, and A. Westerberg, Eds. Contemp. Math. 391, American Mathematical Society, Providence, RI, 2005, 157-165.

[15] N. Kamiya and S. Okubo. On $\delta$-Lie supertriple systems associated with $(\epsilon, \delta)$-Freudenthal-Kantor supertriple systems. Proc. Edinburgh Math. Soc., 43 (2000), 243-260.

[16] N. Kamiya and S. Okubo. Construction of Lie superalgebras $D(2,1 ; \alpha), G(3)$ and $F(4)$ from some triple systems. Proc. Edinburgh Math. Soc., 46 (2003), 87-98.

[17] N. Kamiya and S. Okubo. A construction of simple Lie superalgebras of certain types from triple systems. Bull. Austral. Math. Soc., 69 (2004), 113-123.

[18] N. Kamiya and D. Mondoc. A new class of nonassociative algebras with involution. Proc. Japan Acad. Ser. A, 84 (2008), 68-72. 
[19] S. Kaneyuki and H. Asano. Graded Lie algebras and generalized Jordan triple systems. Nagoya Math. J., 112 (1988), 81-115.

[20] I. L. Kantor. Graded Lie algebras. Trudy Sem. Vect. Tens. Anal., 15 (1970), 227-266.

[21] I. L. Kantor. Some generalizations of Jordan algebras. Trudy Sem. Vect. Tens. Anal., 16 (1972), 407-499.

[22] I. L. Kantor. Models of exceptional Lie algebras. Soviet Math. Dokl., 14 (1973), 254-258.

[23] I. L. Kantor and N. Kamiya. A Peirce decomposition for generalized Jordan triple systems of second order. Comm. Algebra, 31 (2003), 5875-5913.

[24] M. Koecher. Embedding of Jordan algebras into Lie algebras I. Amer. J. Math., 89 (1967), $787-816$.

[25] M. Koecher. Embedding of Jordan algebras into Lie algebras II. Amer. J. Math., 90 (1968), 476-510.

[26] D. Mondoc. Models of compact simple Kantor triple systems defined on a class of structurable algebras of skew-dimension one. Comm. Algebra, 34 (2006), 3801-3815.

[27] D. Mondoc. On compact realifications of exceptional simple Kantor triple systems. J. Gen. Lie Theory Appl., 1 (2007), 29-40.

[28] D. Mondoc. Compact realifications of exceptional simple Kantor triple systems defined on tensor products of composition algebras. J. Algebra, 307 (2007), 917-929.

[29] D. Mondoc. Compact exceptional simple Kantor triple systems defined on tensor products of composition algebras. Comm. Algebra, 35 (2007), 3699-3712.

[30] S. Okubo. Symmetric triality relations and structurable algebras. Linear Algebra Appl., 396 (2005), 189-222.

[31] J. Tits. Une classe d'algèbres de Lie en relation avec les algèbres de Jordan. Nederl. Acad. Wetensch. Proc. Ser. A, 65 (1962), 530-535.

[32] K. Yamaguti and A. Ono. On representations of Freudenthal-Kantor triple systems $U(\epsilon, \delta)$, Bull. Fac. School Ed. Hiroshima Univ., 7 (1984), 43-51.

Received September 01, 2008

Revised December 09, 2008 